\title{
MYCOBACTERIAL SPECIES CAUSING PULMONARY TUBER- CULOSIS AT THE KORLE BU TEACHING HOSPITAL, ACCRA, GHANA
}

\author{
"K.K. ADDO, K. OWUSU-DARKO, D. YEBOAH-MANU, P. CAULLEY ${ }^{1}$, M. MINAMI- \\ KAWA, F. BONSU ${ }^{2}$, C. LEINHARDT ${ }^{3}$, P. AKPEDONU and D. OFORI-ADJEI \\ Bacteriology Department, Noguchi Memorial Institute for Medical Research, P.O. Box LG 581, Le- \\ gon, Ghana, ${ }^{1}$ National Public Health Reference Laboratory, Korle-Bu, Ghana, \\ ${ }^{2}$ National Tuberculosis Control Programme, Korle-Bu, Ghana and \\ ${ }^{3}$ West African TB Research Initiative, Dakar, Senegal
}

\begin{abstract}
SUMMARY
Objective: Characterize mycobacterial species causing pulmonary tuberculosis (PTB) at the Korle-Bu Teaching Hospital in Ghana.

Design: Sputum smear positive samples, two (2) from 70 patients diagnosed as having tuberculosis, after they had consented, were collected from the Korle-Bu Teaching Hospital Chest Clinic between January and July 2003.

Setting: Korle-Bu Teaching Hospital Chest Clinic, Accra.

Results: Sixty-four mycobacterial isolates were obtained and confirmed as members of Mycobacterium tuberculosis complex by colonial morphology and conventional biochemical assays. Fortyseven $(73 \%)$ were $M$. tuberculosis, the human strain, $2(3 \%)$ M. bovis, the bovine strain, 13 (20\%) M. africanum I (West Africa type), and 2 (3\%) M. africanum II (East Africa type).

Conclusion: The results indicate that, there are various strains causing PTB at the Korle-Bu Teaching Hospital and of great concern is $M$. bovis, which mostly causes extra-PTB in humans but found to cause PTB in this study. This calls for the need to conduct a nationwide survey using both conventional and molecular techniques to characterize various mycobacterial species causing TB in Ghana. This will result in better understanding of the various strains circulating in the country and inform individual TB treatment regimen especially the inclusion or exclusion of pyrazinamide.
\end{abstract}

Keywords: $M$. tuberculosis, $M$. africanum, $M$. bovis, Pulmonary Tuberculosis, Bovine Tuberculosis

\section{INTRODUCTION}

Tuberculosis (TB), a disease of great antiquity continues to be a major public health problem worldwide. One-third of the world's over six billion population is infected with the tubercle bacilli with over two million deaths annually ${ }^{1}$.

The available data on TB in Ghana indicate that the disease burden is high and TB remains an important cause of major disability and death in the country ${ }^{2}$. With Ghana's population of over 20 million, The World Health Organization (WHO) estimates 44,041 new cases of all forms of TB in Ghana corresponding to a TB incidence rate of 211 per 100,000 inhabitants of whom 19,285 are smear positive cases ${ }^{3}$.

This upsurge of TB is attributed to factors such as HIV/AIDS, poverty, population growth, overcrowding, malnutrition, stress, drugs and alcohol abuse, consumption of contaminated meat and milk and multi-drug resistant (MDR) TB.

TB is known to be caused mainly by the mammalian tubercle bacilli, Mycobacterium tuberculosis complex, which consists of closely related species. The species are M. tuberculosis, M. bovis, M.bovis Bacillus Calmette-Guérin (BCG), M. africanum, M. microti, M. canetti and M. pinnipedil ${ }^{4,5,6}$. M. tuberculosis principally causes TB in humans but can affect animals that have contact with infected humans. It causes mainly pulmonary type of TB but extra-pulmonary type has also been reported. M. bovis which causes bovine TB affects cattle, humans, sheep, goats, pigs and several other domestic and wild animals. M. bovis BCG on the other hand is termed the vaccine strain and can cause disseminated BCG infection in vaccinated children ${ }^{7}$. M. africanum is considered to be an in-

* Author for correspondence

kaddo@noguchi.mimcom.net 
termediate species between $M$. tuberculosis and $M$. bovis, being closer to the latter ${ }^{8}$. The organism causes TB in humans and occasionally animals such as apes ${ }^{9}$. M. microti which is attenuated in humans is a member of the complex and termed the vole bacillus as it causes TB in voles (Microtus agrestis) and other small rodents such as hamsters, shrews, rats, mice, rabbits and guinea-pigs ${ }^{10} . M$. canetti is rarely seen but can cause TB in humans ${ }^{5}$ whereas $M$. pinnipedii is described as the seal bacillus for causing TB in fish-eating sea animals ${ }^{6}$.

Differentiation within the complex is necessary for epidemiological purposes and some individual patient treatment ${ }^{11}$. However, in Ghana as in most developing countries, TB is mostly diagnosed by sputum smear microscopy (SSM) which cannot differentiate members of the complex. Routine acid-fast bacilli (AFB) culture and identification of isolates are not performed due to lack of well equipped laboratory facilities. With the establishment of biosafety level 3 (P3) mycobacterial laboratory at Noguchi Memorial Institute for Medical Research (NMIMR), a study was initiated in 2003 with the main objective of characterizing mycobacterial species causing pulmonary tuberculosis in patients attending the Korle-Bu Teaching Hospital Chest Clinic in Accra, Ghana. This was after ethical clearance was granted by the Institutes' ethical review board on research.

\section{MATERIALS AND METHODS \\ Sample Collection}

Sputum smear positive samples, two (2) from each patient diagnosed as having tuberculosis, after they had consented were collected from the Chest Clinic of the Korle-Bu Teaching Hospital (KBTH) which acts as national referral clinic, between January and July 2003. Samples were taken from seventy patients into sterile plastic containers and transported immediately to the Bio-safety Level 3 (P3) TB Laboratory at NMIMR for processing: microscopy, culture and identification.

\section{Sputum Smear Microscopy}

To be certain that the samples were positive; sputum smear examination was performed once again by the Ziehl-Neelsen technique: $0.3 \%$ carbol fuchsin for $5 \mathrm{~min}, 20 \%$ sulphuric acid as a decolouriser for $5 \mathrm{~min}$ and $0.3 \%$ methylene blue as a counterstain for $1 \mathrm{~min}$.

\section{Culture of Sputum Samples Culture Medium}

Two percent $(2 \%)$ modified Ogawa egg medium (Kudoh medium) (which has an advantage over
Lowenstein-Jensen medium in developing countries as centrifugation is not required) was prepared according to Ogawa and $\mathrm{Saba}^{12}$ and Kudoh and $\mathrm{Kudoh}^{13}$. Since medium-containing glycerol favours the growth of $M$. tuberculosis but inhibits the growth of both $M$. bovis and $M$. africanum, another set of medium without glycerol but containing $0.5 \%$ sodium pyruvate, which encourages the growth of $M$. bovis and $M$. africanum, was also prepared $^{4}$.

\section{Pre-treatment of Sputum Sample}

One volume of $4 \%$ sodium hydroxide - a decontaminant, was added to 1 volume of the sputum sample and stirred with pipette to homogenize. In cases of heavy mucous sample, it was left for about 10 minutes to digest after the addition of the decontaminant.

\section{Inoculation, Incubation and Reading}

About $0.1 \mathrm{ml}$ of the pre-treated sample was inoculated into 4 tubes: 2 tubes containing glycerol and the other 2 containing pyruvate. The inoculated slants were incubated at $37^{\circ} \mathrm{C}$.

The culture tubes were observed one week after incubation for rapid growers and at 4 weeks for slow growers. When visible colonies did not appear at 4th week, observation continued weekly until 8 weeks before declaring the culture negative. The growth that appeared as colony in the 4th week, was recorded as rough or smooth, eugonic or dysgonic, buff, white, yellow or orange. The actual numbers of colonies seen and counted were also recorded as well as contaminated tubes.

\section{Identification Tests}

Besides morphological observations, the following biochemical tests were performed on the isolates. All the identification tests were carried-out on subcultured strains using fresh Ogawa slants.

\section{Para-Nitrobenzoic Acid (PNB) Susceptibility Test}

This was performed according to Tsukamura ${ }^{14}$ to differentiate members of $M$. tuberculosis complex from other mycobacterial strains. In brief, $0.1 \mathrm{ml}$ of bacillary suspension prepared from 3-week old sub-culture was inoculated into 2 tubes of $0.5 \mathrm{mg} / \mathrm{ml}$ PNB-containing and PNB-free media and incubated at $37^{\circ} \mathrm{C}$ for 4 to 28 days. The tubes were observed on the $4^{\text {th }}, 7^{\text {th }}, 14^{\text {th }}, 21^{\text {st }}$, and $28^{\text {th }}$ days for visible growth of colonies or otherwise. No growth observed in any of the PNB containing tubes after the 28 days of incubation was indicative of members of the $M$. tuberculosis complex. 


\section{Niacin Accumulation Test}

BBL Taxo TB Niacin Test Strips (Becton and Dickinson, USA), absorbent paper strips and TB Niacin Positive Test Control paper discs were used according to the manufacturers' instruction. Fourweek old sub-cultures having at least 50-100 colonies were used.

About $2.5 \mathrm{ml}$ sterile distilled water was added to the culture and the surface growth gently punctured using $1 \mathrm{ml}$ sterile pipette to permit extraction of niacin. With the help of sterile transfer pipette, approximately $0.6 \mathrm{ml}$ of the fluid extract was carefully removed and transferred to the bottom of 20x125 mm screw cap test tube. Negative control was also prepared. The strips were dropped with arrow downward into the tubes: positive and negative controls, test culture and stopper immediately. The colours of the extracts were then compared after 15 minutes. Niacin accumulation was indicated by vivid appearance of a yellow colour in the extract.

\section{Nitrate Reduction to Nitrite Test}

BBL Taxo TB Nitrite Test Strips (Becton and Dickinson, USA), absorbent paper strips were also used according to the manufacturers' instruction. Four-week old sub-cultures were used. About 0.5 $\mathrm{ml}$ sterile distilled water was transferred to each test tube. Using a sterile $1 \mathrm{ml}$ pipette, two clumps of growth were removed from the culture tube and added to the distilled water and dispersed with the pipette. The strips were then transferred into the tubes and incubated at $37^{\circ} \mathrm{C}$ for 2 hours. The colours of the top portion of the strips were noted immediately after the incubation. Positive and negative controls tests were also performed following the same procedure. Nitrate reduction to nitrite, was indicated by change of colour of the top portion of the test strip from white to dark blue.

\section{Thiophene Carboxylic Acid Hydrazide (TCH) Susceptibility Test}

This was performed according to Yates and Grange $^{15}$. About $0.1 \mathrm{ml}$ of bacillary suspension prepared from 3-week old sub-culture was inoculated into 2 tubes of $0.5 \mathrm{mg} / \mathrm{ml} \mathrm{TCH}$-containing and $\mathrm{TCH}$-free Ogawa media and incubated at $37^{\circ} \mathrm{C}$ for 3 to 4 weeks. The tubes were observed on the $4^{\text {th }}, 7^{\text {th }}, 14^{\text {th }}, 21^{\text {st }}, 28^{\text {th }}$ days for visible growth of colonies indicating resistance or otherwise.

\section{Oxygen Preference Test}

Middlebrook $7 \mathrm{H} 9$ media rendered semisolid by addition of $0.1 \%$ agar were used according to
Marks $^{16}$. The test was performed to distinguish microaerophilic bacilli from strictly aerobic ones. About $0.2 \mathrm{ml}$ of bacillary suspension prepared from 3 - week old sub-culture was inoculated about $1 \mathrm{~cm}$ below the media surface. The tubes were incubated at $37^{\circ} \mathrm{C}$ undisturbed for 18 days. Aerobic growth occurred near the surface of the culture tubes, extending about $5 \mathrm{~mm}$ below it whereas microaerophilic growth occurred as a band, about $10-20 \mathrm{~mm}$ below the surface.

\section{Pyrazinamide (PZA) Susceptibility Test}

The procedure according to Wayne ${ }^{17}$ was followed. In brief, 2 loopfuls of 3-week old subculture colonies were inoculated into two Middlebrook $7 \mathrm{H} 11$ agar containing $0.1 \mathrm{mg} / \mathrm{ml}$ PZA and $2 \mathrm{mg} / \mathrm{ml}$ sodium pyruvate and incubated together with 2 control tubes at $37^{\circ} \mathrm{C}$ for 4 days. Afterwards, $1 \mathrm{ml}$ of freshly prepared solution of ferrous ammonium sulphate, $1 \% \mathrm{w} / \mathrm{v}$ in distilled water was added to each of the test and control media and left at room temperature for 30 minutes and results read.

Pyrazinamidase positive was indicated by a pink band at the upper part of the butt of the tubes.

\section{RESULTS}

All the sputum samples were confirmed as AFB positive by SSM. With respect to culture, on the average, growth appears within 3-4 weeks of incubation confirming the strains as slow growers. Of the 70 patients sample cultured, growth occurred in 64 of them, giving a recovery rate of $91 \%$; of the remaining 6 samples, contamination was observed in 3 and no growth declared in another 3 after 8 weeks of incubation (Table 1).

The colonial morphologies of forty-nine (49) isolates were dry and rough with irregular margins, typical eugonic growth with buff pigmentation, all suggestive of $M$. tuberculosis or $M$. africanum type II. The morphologies of thirteen (13) isolates were smooth and dysgonic with buff pigmentation, suggestive of $M$. africanum $\mathrm{I}$. The colonies of the remaining two (2) isolates were smooth and dysgonic with white pigmentation, suggestive of $M$. bovis.

Enhanced growth of M. tuberculosis was observed in medium containing glycerol whereas appreciable growth of $M$. bovis and $M$. africanum was observed in pyruvate-containing medium. 
Table 1 Pattern of sputum growth and contamination $(\mathrm{n}=70)$

\begin{tabular}{ccl}
\hline Grading & $\begin{array}{c}\text { Number of } \\
\text { Samples }\end{array}$ & \multicolumn{1}{c}{$\begin{array}{c}\text { Number of Colonies } \\
\text { Counted }\end{array}$} \\
\hline Negative & 3 & No Growth \\
Positive & 5 & Between 1-19 Colonies \\
$1+$ & 30 & Between 20-100 Colonies \\
$2+$ & 25 & Between 100-200 Colonies \\
$3+$ & 2 & Between 200-500 Colonies \\
$4+$ & 2 & $\begin{array}{l}\text { More than 500 Colonies } \\
\text { Quarter of the medium } \\
\text { contaminated } \\
\text { C1 }\end{array}$ Half of the medium Con- $^{\text {taminated }}$ \\
C2 & 2 &
\end{tabular}

The identification of the isolates were confirmed by the biochemical assays as shown in summary in Table 2. found in East Africa was found in $2(3 \%)$ of the isolates.

Although this study reports on characterization of only sixty-four isolates, the results reveal that there are various strains of mycobacteria causing pulmonary tuberculosis in Ghana. This calls for typing of isolates as in addition to epidemiological importance, it may be useful for individual patient treatment as some of the strains such as M. bovis are naturally resistant to pyrazinamide ${ }^{18}$, one of the first-line anti-tuberculosis drugs used in the country. Previous studies on characterization of mycobacterial species causing pulmonary tuberculosis in Ghana and some West African countries indicated the same trend but $M$. bovis was rarely isolated. Van der Werf et al. ${ }^{19}$ looking at speciation of 99 isolates from TB patients presenting at the Chest Clinic in the middle belt of Ghana found the

Table 2 Characterization of Mycobacterial Isolates $(n=64)$

\begin{tabular}{|l|c|c|c|c|c|c|c|}
\hline \multicolumn{1}{|c|}{ Strain } & No. (\%) & PNB & Niacin & Nitrate & TCH & Oxygen & PZA \\
\hline M. tuberculosis & $47(73)$ & - & + & + & Res & Aer & Sus \\
M. bovis & $2(3)$ & - & - & - & Sus & Mic & Res \\
M. africanum I & $13(20)$ & - & weak + & - & Sus & Mic \\
M. africanum II & $2(3)$ & - & weak + & + & Sus & Mic & Sus \\
\hline
\end{tabular}

Key: No. = number of strains; $\mathrm{PNB}=$ susceptibility to para-nitrobenzoic acid; Niacin = niacin accumulation; Nitrate = nitrate reduction to nitrite; TCH = susceptibility to thiophene carboxylic acid hydrazide; Oxygen = oxygen preference; PZA = susceptibility to pyrazinamide; - = negative; $+=$ positive; Res $=$ resistant; Sus $=$ Susceptible; Aer $=$ aerobic; Mic $=$ microaerophilic.

\section{DISCUSSION}

The present study confirmed the general observation that TB is caused mainly by the members of the $M$. tuberculosis complex as $47(73 \%)$ of the isolates were found to be M. tuberculosis, 2 (3\%) M. bovis, $13(20 \%)$ M. africanum type I and 2 (3\%) M. africanum type II. M. bovis BCG strain was not among the isolates confirming the notion that its isolation is more common in children than adults who were the present study subjects. The results have also incriminated $M$. tuberculosis as the most prevalent causative agent of pulmonary $\mathrm{TB}$ at the Korle-Bu Teaching Hospital. The appreciable number of isolates 13/64 (20\%) found to be $M$. africanum type I support the assertion that this strain which phenotypically resembles $M$. bovis is commonly found in West Africa ${ }^{8}$. The type II which resembles $M$. tuberculosis and principally presence of $M$. tuberculosis in $57(57.6 \%)$ of the isolates and $42(42.4 \%)$ were $M$. africanum. Lawn et $\mathrm{al}^{20}$. also reported speciation of 26 isolates as $M$. tuberculosis $24(92 \%)$ and $M$. africanum 2 (8\%). The two studies reported no $M$. bovis being isolated. Dosso et al. ${ }^{21}$ in a nationwide survey in neighbouring Cote d'Ivoire in 1995-1996 confirmed the involvement of M. tuberculosis in 299 isolates out of $320(93.4 \%)$ and $21(6.6 \%)$ as $M$. africanum. Here too $M$. bovis was not isolated. However, Niobe-Eyangoh et al. ${ }^{22}$ in Quest Province of the Central African country of Cameroon analyzed $455 M$. tuberculosis complex strains and had 41 (9\%) being M. tuberculosis, 413 (90.8\%) being $M$. africanum and $1(0.2 \%)$ being $M$. bovis. None of the above-mentioned studies did characterize $M$. africanum to type I and II. 
The present report is the only study where $M$. bovis has been isolated in Ghana from pulmonary TB patients and attempt also made to type $M$. africanum. Cases caused by $M$. bovis have been associated with extra-pulmonary $\mathrm{TB}^{23}$. Humans are infected by the organism from cattle and other ruminants kept for meat and milk production ${ }^{10}$. Cattle are mostly reared in the coastal and guinea savannah zones of the country where the prevalence of bovine TB is reportedly high ${ }^{23}$. In some of the communities, members live in close contact with their animals that may be infected. Human-tohuman transmission of $M$. bovis is limited and anecdotal as it is believed that pulmonary patients infected by $M$. bovis are less infectious as they eliminate fewer bacilli in their sputum than those infected by $M$. tuberculosis ${ }^{24}$. Although the study could not have data on occupation of patients as it is not routinely recorded in health facilities in Ghana, it is highly recommended to capture such data as farmers, veterinarians and slaughterhouse workers who are exposed to infected animals and handle lesioned carcases are at a higher risk of acquiring $M$. bovis infection through the aerogenous or respiratory route.

TB microscopy is the routine diagnostic tool used in Ghana to diagnose TB and once acid-fast bacilli are found treatment is initiated without any further assays. It is now imperative that a nationwide survey is conducted in all the ecological zones using both conventional and molecular techniques to characterize the various mycobacterial species causing both pulmonary and extra-pulmonary TB and also equip the four public health laboratories in the country to conduct mycobacteria culture and speciation. This will result in better understanding of the circulating strains in the country, inform individual TB treatment regimen especially the inclusion or exclusion of pyrazinamide ${ }^{11}$, and lead to effective National TB Control Programme.

\section{ACKNOWLEDGEMENT}

We would like to acknowledge the Infectious Diseases Project of the Noguchi Memorial Institute for Medical Research (NMIMR) funded by the Japan International Cooperation Agency (JICA) and the Department for International Development (DFID) (contract number AG 2071) as part of the West African TB Research Initiative for their support of this study. We are also grateful to Dr. Sampson Aboagye, Head, Chest Clinic, KBTH and Samuel Kudzawu, Head, Chest Clinic Laboratory, KBTH.

\section{REFERENCES}

1. World Health Organization, Global Tuberculosis Control Report 2000.

2. National Tuberculosis Report. Ghana Health Service/Ministry of Health-TB Five-Year Strategic Plan 2001.

3. World Health Organization, Global Tuberculosis Control Report 2005.

4. Grange JM, Yates MD, De Kantor, IN. Guidelines for speciation within the Mycobacterium tuberculosis complex. WHO/EMC/ZOO/96/4 1996.

5. Van Soolingen D, Hoogenboezem T, de Haas PE, Hermans PW, Koedam MA, Teppema KS. A novel pathogenic taxon of Mycobacterium tuberculosis complex, Canetti: characterization of an exceptional isolate from Africa. Int J Syst Bacteriol 1997; 47: 1236-1245.

6. Cousins D, Bastida R, Cataldi A, Quse V, Redrobe S, Dow S. Tuberculosis in seals caused by a novel member of the Mycobacterium tuberculosis complex: Mycobacterium pinnipedii sp. nov. Int J Syst Evol Microbiol 2003; 53: 1305-1314.

7. Romanus V, Svensson A, Hallender HO. The impact of changing BCG coverage on tuberculosis incidence in Swedish born children between 1969 and 1989. Tubercle Lung Disease 1992; 73: 150-161.

8. Grange JM, Yates MD. Incidence and nature of human tuberculosis due to $M$. africanum in South-East England 1977-1987. Epidem Inf 1989; 103: 127-132.

9. Thorel, M.F., 1980. Isolation of M. africanum from monkeys. Tubercle 1980: 61: 101-104.

10. Moda G, Daborn CJ, Grange JM, Cosivi O. The zoonotic importance of Mycobacterium bovis. Review article. Tubercle and Lung Disease 1996; 77: 103-108.

11. Parsons LM, Brosch R, Cole ST, Somoskovi A, Loder A, Bretzel G. Rapid and simple approach for identification of Mycobacterium tuberculosis complex isolates by PCR-based genomic deletion analysis. J Clin Microbiol 2002; 2339-2345. 
12. Ogawa T, Saba, K. On the quantitative cultivation of tubercle bacilli. Kekkaku 1949; 2: 13-29.

13. Kudoh S, Kudoh T. A simple technique for culturing tubercle bacilli. Bull WHO 1974; 51: 71-82.

14. Tsukamura M, Tsukamura S. Differentiation of Mycobacterium tuberculosis and Mycobacterium bovis by p-nitrobenzoic acid susceptibility. Tubercle 45: 64-65.

15. Yates MD, Grange JM. A study of the relationship between the resistance of Mycobacterium tuberculosis to isonicotinic acid hydrazide (isoniazid) and to thiophen-2-carboxylic acid hydrazide. Tubercle 65: 295-299.

16. Marks J. A system for the examination of tubercle bacilli and other Mycobacteria. Tubercle 57: 207-225

17. Wayne LG. Simple pyrazinamide and urease tests for routine identification of Mycobacteria. Am Rev Respir Dis 109: 147-151.

18. WHO/IUATLD. Anti-Tuberculosis Drug Resistance in the World. The Global Project on Anti-TB Drug Resistance Surveillance (Document, WHO/TB/1997.229) 1997.

19. Van der Werf TS, Groothuis DG, van Kingeren B. High initial drug resistance in pulmo- nary tuberculosis in Ghana. Tubercle 1989; 70: 249-255.

20. Lawn SD, Frimpong $\mathrm{EH}$, Al-Ghusein $\mathrm{H}$, Acheampong JW, Uttley, AHC, Butcher PD, Griffin GE. Pulmonary tuberculosis in Kumasi, Ghana: presentation, drug resistance, molecular epidemiology and outcome of treatment. West Afri J of Med 2001; 20: 92-97.

21. Dosso M, Bonard D, Msellati P, Doulhourou C, Vincent V, Peyre M, Traore M, Koffi K, Coulibaly IM. Primary resistance to antituberculosis drugs: a national survey conducted in Cote d'Ivoire in 1995-1996. Intl J of Tubercus and Lung Dis 1999; 9: 805-809.

22. Niobe-Eyangoh SN, Kuaban $\mathrm{C}$, Sorlin $\mathrm{P}, \mathrm{Cu}-$ nin $\mathrm{P}$, Thonnon J, Sola C, Rastogi N, Vincent V, Gutierrez MC. Genetic Biodiversity of $M y-$ cobacterium tuberculosis Complex Strains with Pulmonary Tuberculosis in Cameroon. $J$ of Clin Microbiol 2003: 41: 2547-2553.

23. Bonsu OA, Laing E, Akanmori BD. Prevalence of tuberculosis in the Dangme-West district of Ghana, public health implications. Acta Tropica 2000; 76: 9-14.

24. Kovalyov GK. On the human tuberculosis due to Mycobacterium bovis. A review. Microbiol Immunol 1989; 33: 199-206. 